\title{
Measurement of strain in Al-Cu interconnect lines with x-ray microdiffraction
}

\author{
H. H. Solak, a) Y. Vladimirsky, and F. Cerrina \\ Department of Electrical and Computer Engineering, University of Wisconsin-Madison, Madison, \\ Wisconsin 53706
}

B. Lai, W. Yun, Z. Cai, P. Ilinski, D. Legnini, and W. Rodrigues

Argonne National Laboratory, Argonne, Illinois 60439

(Received 9 March 1999; accepted for publication 14 April 1999)

\begin{abstract}
We report measurement of strain in patterned $\mathrm{Al}-\mathrm{Cu}$ interconnect lines with x-ray microdiffraction technique with $\mathrm{a} \sim 1 \mu \mathrm{m}$ spatial resolution. Monochromatized $\mathrm{x}$ rays from an undulator were focused on the sample using a phase fresnel zone plate and diffracted light was collected by an area detector in a symmetric, angle dispersive x-ray diffraction geometry. Measurements were made before and after the line sample was stressed for electromigration. Results show an increase in interand intra-grain strain variation after the testing. Differences in strain behavior of grains with (111) and (200) crystallographic planes parallel to the substrate surface were observed. A position dependent variation of strain after the testing was measured whereas no such dependence was found before the testing. (C) 1999 American Institute of Physics. [S0021-8979(99)08214-6]
\end{abstract}

\section{INTRODUCTION}

Mechanical stress in $\mathrm{Al}$ based interconnect lines in integrated circuits has attracted much attention recently because of its role in circuit reliability. Al metallization films are usually left in tensile stress after manufacturing processes at elevated temperatures due to the differential contraction of $\mathrm{Al}$ and the surrounding substrate and passivation materials. Circuit failures were observed due to stress induced voiding that sever connection in these lines. ${ }^{1,2}$

Electromigration is the movement of conductor atoms under the influence of an electrical current. The electron wind force arising due to the momentum transfer from the drifting electrons is responsible for the migration. ${ }^{3}$ High current densities $\left(\sim 10^{5} \mathrm{~A} / \mathrm{cm}^{2}\right)$ found in interconnects create large enough mass transport to create voids and extrusions (hillocks) in lines causing circuit failures. Electromigration is a major interconnect reliability problem that interacts strongly with mechanical stress present in interconnects. It has been intensely researched since its early identification as a device failure mechanism ${ }^{4}$ partly due to the relentless miniaturization trends in the microelectronic circuits that push higher current densities on the interconnects exacerbating the electromigration problem.

As the transport of material due to electromigration proceeds in a metal line material is accumulated near the anode end and depleted near the cathode end. This creates a stress gradient along the line which is confined by the substrate and the dielectric passivation layer. This induced stress field creates a chemical potential gradient opposing the electromigration force. Therefore, atoms start feeling two opposing forces, one due to the electron wind and the other one due to the stress gradient. Under certain conditions these two forces can completely balance each other and completely halt the

\footnotetext{
${ }^{a)}$ Electronic mail: solak@xraylith.wisc.edu
}

atomic flow. Under the steady-state balance conditions a constant stress gradient is exists along the line. I. Blech has shown that there is a critical interconnect length electric current density product under which this balance is achieved and no electromigration damage is observed. ${ }^{5}$ This phenomenon significantly affects the resistance of interconnect structures to electromigration damage.

Models have been developed to predict the evolution of stress in interconnects due to electromigration. ${ }^{6-10}$ However, experimental verification has proved more difficult mainly because of the difficulty of stress measurement with micron or submicron scale spatial resolution. Indirect measurements have concentrated on measurement of stress through its effects on the substrate material such as Raman spectroscopy ${ }^{11}$ and transistor gain modification. ${ }^{12} \mathrm{X}$-ray diffraction is a direct technique for stress measurement and has been successfully used for detailed characterization of average stress in interconnect lines. ${ }^{13-15}$ However, the sampled area with traditional $\mathrm{x}$-ray tools is too large to allow measurement of stress distribution with necessary spatial resolution. Development of extremely high brightness synchrotron radiation sources such as the advance photon source (APS) and advances in $\mathrm{x}$-ray optics such as the fresnel zone plates (FZP) for hard $\mathrm{x}$-ray radiation has opened new possibilities in this area.

Recently, Wang et al. have used tapered glass capillary optics and pinholes to perform x-ray diffraction experiments on $10 \mu \mathrm{m}$ wide $\mathrm{Al}$ lines with approximately $10 \mu \mathrm{m}$ resolution. ${ }^{16}$ White light from a synchrotron was used in this energy dispersive diffraction study. Their results showed a stress gradient build up in the line as electromigration proceeded. They used Blech's and Korhonen's models successfully to explain the steady state and transient stress behavior, respectively. Although this experiment confirms the predictions of the models for relatively wide lines, the situation in fine lines remains to be addressed experimentally. In fact, 


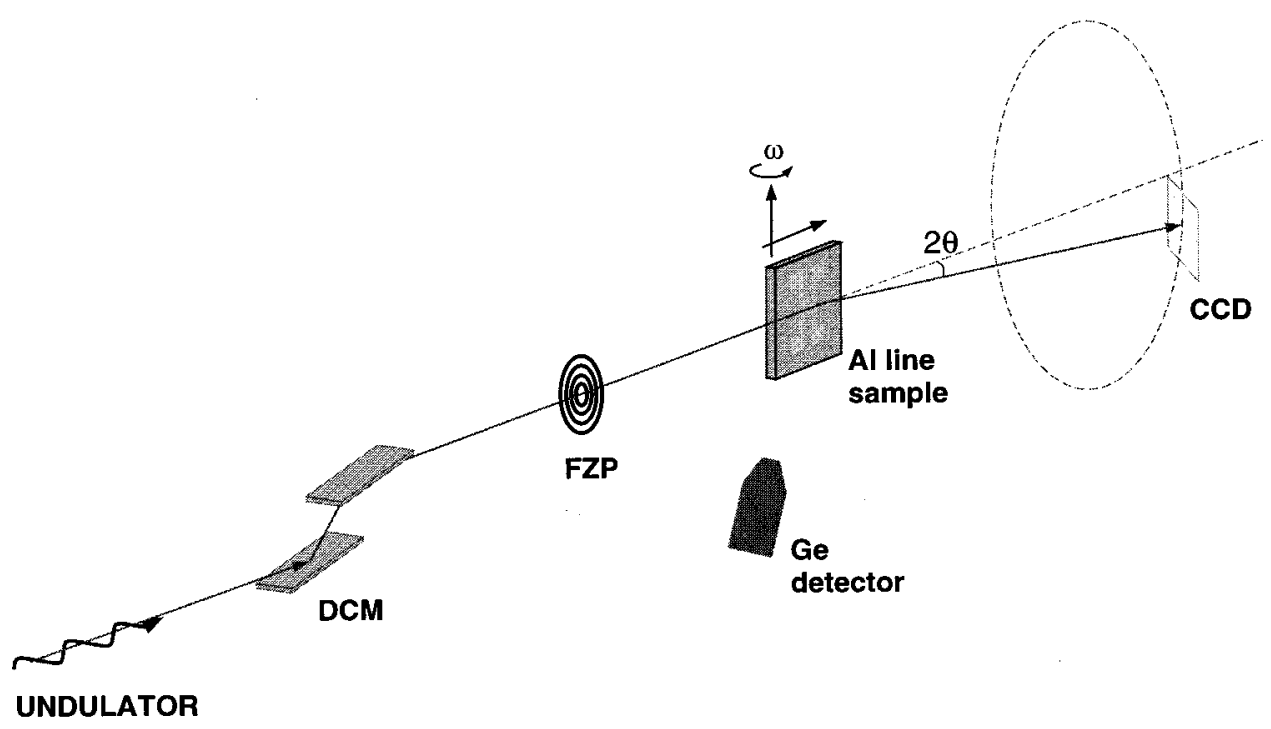

FIG. 1. Schematic view of the setup for microdiffraction experiment. X rays produced by an undulator in the storage ring are monochromatized by a DCM and focused on the thin Al-Cu film sample by a FZP. The sample is mounted on a goniometer with scanning stage. The patterned Al-Cu line runs perpendicular to the horizontal plane. The CCD camera is mounted on the $2 \theta$ arm of the goniometer in the horizontal plane. Energy dispersive Ge detector receives fluorescent $\mathrm{x}$ ray from the sample. All the components shown in the figure after the DCM are mounted on the same optical table for vibration isolation.

line width is expected to strongly affect the threedimensional stress state of the line as aspect ratios continue to grow. For example, the equi-biaxial stress approximation adopted in Wang's work does not hold for narrower lines as was reported by Besser. ${ }^{15}$

\section{EXPERIMENT}

We conducted experiments on the 2-ID-D undulator beamline of APS $^{17,18}$ using FZP as focusing elements for measuring strain distribution on $\mathrm{Al}-\mathrm{Cu}$ interconnects. The zone plates were manufactured at the Center for X-ray Lithography by $\mathrm{x}$-ray replication and $\mathrm{Au}$ electroplating techniques. ${ }^{19}$ The experimental station is equipped with a goniometer, scanning sample-stage with submicron resolution, an energy dispersive Ge x-ray detector and an x-ray charge coupled device (CCD) camera along with other optical alignment instruments. The $\mathrm{Si}$ (111) double-crystal monochromator $(\mathrm{DCM})$ in the beamline provides $\mathrm{x}$ rays with a bandwidth of $\Delta \lambda / \lambda=2 \times 10^{-4}$. A beam spot size of 0.2 $\mu \mathrm{m}$ was achieved on this beamline but that level of spatial resolution was not deemed necessary for this experiment.

The interconnect line sample was a single $1.6 \mu \mathrm{m}$ wide, $270 \mu \mathrm{m}$ long passivated $\mathrm{Al}-2 \% \mathrm{Cu}$ line. The stack consisted of $\mathrm{Si} / \mathrm{SiO}_{2}(200 \mathrm{~nm}) / \mathrm{Al}-2 \% \mathrm{Cu}(600 \mathrm{~nm}) / \mathrm{SiO}_{2}(700 \mathrm{~nm})$. Samples were manufactured using standard semiconductor processing procedures. The thin film line was connected to four contact pads for electrical testing. The sample was annealed at $450{ }^{\circ} \mathrm{C}$ for $30 \mathrm{~min}$ in forming gas after patterning. Another examined sample was an unpatterned 2.5 - $\mu \mathrm{m}$-thick Al$0.5 \% \mathrm{Cu}$ film deposited on a $\mathrm{Si} / \mathrm{SiO}_{2}$ substrate. This sample was annealed under same conditions but no passivation layer was deposited on top. This thick film sample was used as a reference in the calibration of diffraction data. We have not confirmed the strain free state of this film, but nevertheless used it as a reference in our experiment in which measure- ment of relative changes in strain along the line was the major concern rather than the absolute strain levels.

Figure 1 shows a schematic view of the experimental setup. $9.5 \mathrm{keV}$ photons were incident on the sample at a $16^{\circ}$ incidence angle. The CCD detector was placed at an angle of $32^{\circ}$ to accept both (111) and (200) reflections from the $\mathrm{Al}$ lattice in an approximately symmetric reflection geometry. These reflections originate from grains in the polycrystalline thin film sample that have (111) and (200) lattice planes approximately parallel to the substrate surface. In the following we will simply refer to these two distinct set of grains as (111) and (200) types respectively. The interconnect line was placed in the focused beam by following the $\mathrm{Cu} K \alpha$ fluorescent emission which was detected by the energy dispersive $\mathrm{x}$-ray detector. The incident $\mathrm{x}$-ray beam size along the line direction was $0.8 \mu \mathrm{m}$ illuminating a same length section of the $1.6 \mu \mathrm{m}$ wide line. The sample was rocked in the $\omega$ circle in $\pm 1.5^{\circ}$ range in order to increase the number of reflections detected from the grains of the Al line. A sample CCD image obtained from the line sample showing (111) and (200) diffraction spots is shown in Fig. 2.

After the line sample was placed in the focused x-ray beam, microdiffraction images were obtained from a $100 \mu \mathrm{m}$ long section of the line at $2-4 \mu \mathrm{m}$ intervals. The sample was subsequently removed from the goniometer stage and placed in an oven for electromigration stressing. During the accelerated testing an electric current of $18 \mathrm{~mA}$ was passed for approximately $3.5 \mathrm{~h}$ at $300{ }^{\circ} \mathrm{C}$. The resistance of the line increased by $\sim 1 \%$ during this time presumably due to electromigration damage. Same $100 \mu \mathrm{m}$ section of the line was again inspected using microdiffraction technique for changes in the stress state. Microdiffraction data from the thick blanket film sample was obtained in the same diffraction geometry but no thermal testing was performed on this sample. 


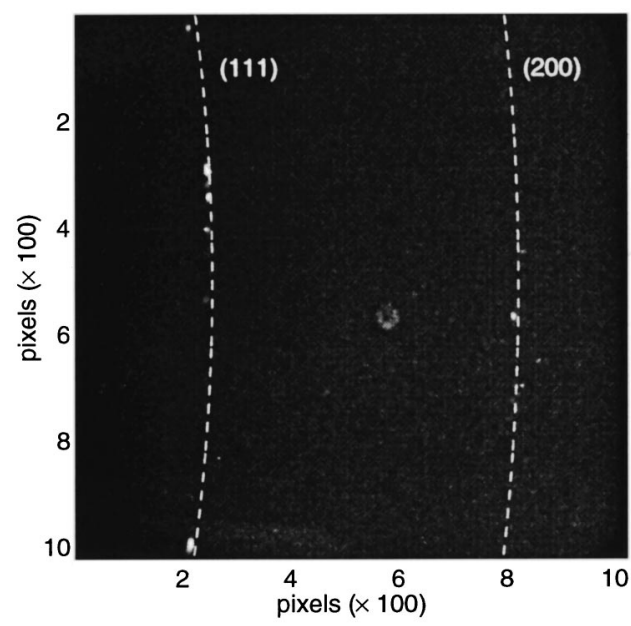

FIG. 2. A typical microdiffraction image file showing several (111) and (200) diffraction spots. The dashed lines show the calculated diffraction rings expected from a strain-free $\mathrm{Al}$ sample.

\section{RESULTS AND DISCUSSIONS}

Figure 3 shows the microdiffraction pattern obtained from the thick $(2.5 \mu \mathrm{m}) \mathrm{Al}-0.5 \% \mathrm{Cu}$ film. The bright rings in this image are due to the (111) and (200) reflections as marked in the figure. The image resembles a powder diffraction pattern with almost continuous diffraction rings. The spottiness is due to reflections from individual grains in the film. The footprint of the microbeam illuminates an $\sim 8 \mu \mathrm{m}^{2}$ area on the sample due to the small angle of incidence. Considering the three-dimensional microstructure of the thick film and $0.5 \mu \mathrm{m}$ average grain size we can estimate the number of grains in the illuminated volume to be in the order of several hundreds. Therefore, it is not surprising that a large number of spots $(\sim 40-50)$ were obtained from this sample especially because of the fact that the sample was rocked within a $\pm 1.5^{\circ}$ range in the $\omega$ circle during data acquisition. The presence of the (200) reflection spots in large numbers indicates a lack of (111) texture in this sample which was also the case with the patterned thin film samples.

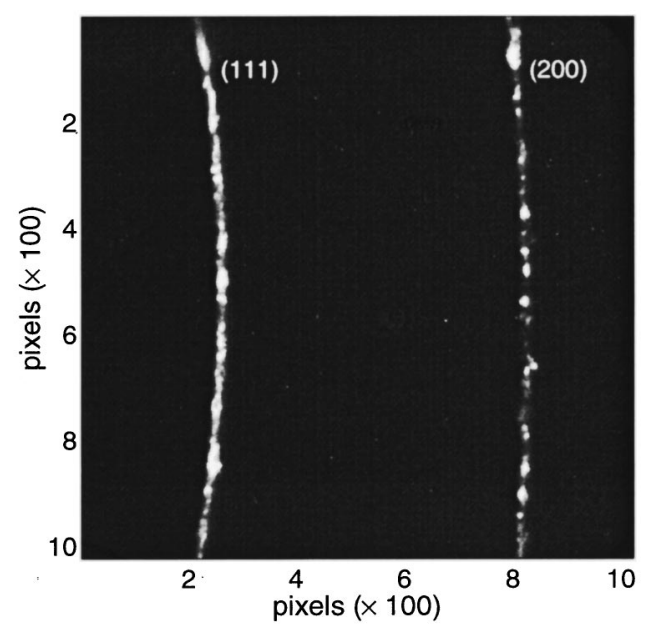

FIG. 3. Microdiffraction pattern from the 2.5- $\mu$ m-thick unpatterned Al$0.5 \% \mathrm{Cu}$ film. The bright and spotty rings are due to (111) and (200) reflections.
The microdiffraction pattern in Fig. 2 obtained from the $1.6 \mu \mathrm{m}$ wide line has only several diffraction spots due to the small number of grains illuminated. The peak positions of the diffraction spots in the two-dimensional diffraction data were obtained with the help of a computer program. Figure 4 shows the collection of those data points for (111) and (200) reflections from the sample line both before and after electromigration. We should emphasize that the peak positions marked in those plots are obtained from a large number of data files, each similar to Fig. 2 resulting from diffraction at different points along the line.

\section{A. Determination of Strain}

The $2 \theta$ angles of diffraction corresponding to the determined peak positions were calculated using the experimentally measured parameters such as the sample-detector distance and the detector angle with straight forward geometrical derivation. The measured lattice spacing is given by the Bragg's equation

$$
d=\frac{\lambda}{2 \sin \theta} .
$$

The dashed lines in Fig. 4 show the "expected" reference diffraction rings from an unstrained $\mathrm{Al}$ lattice calculated from standard measurements. The experimentally found spots also form a ring that is near but somewhat different than the reference one. A mere shift between the measured and calculated rings can be due to calibration errors as well as a real difference of lattice parameter between the two experiments. This difference can be due to reasons such as a nonzero average strains in our sample and impurity and defects. On the other hand, the clear difference in the shape of the two rings is found to be due to the distortions introduced by the area detector. The detector had fiberoptic demagnification optics which is known to be prone to distortions.

Our main focus in this experiment was to measure variations in strain along the line rather than an absolute measurement of average strain, which can be measured quite precisely with large area methods. ${ }^{13-15}$ With this goal in mind we were able to eliminate the effects of the detector distortion by using the data from the thick film (Fig. 3) as our reference diffraction ring. In other words, we used deviations of the measured spot positions from that reference ring to calculate the corresponding strains. Justification of this method is based on the fact that the distortions are slowly varying function of position on the detector surface. Hence,

TABLE I. Average and standard deviations of strain measurements on thick $\mathrm{Al}$ film and the $1.6 \mu \mathrm{m} \mathrm{Al}-\mathrm{Cu}$ line before and after electromigration stressing.

\begin{tabular}{lcc}
\hline \hline & $\bar{\epsilon}\left(\times 10^{-3}\right)$ & $\sigma_{\epsilon}\left(\times 10^{-3}\right)$ \\
\hline Thick Al [111] & 0 & 0.69 \\
Thick Al [200] & 0 & 0.86 \\
Al line before [111] & 2.6 & 0.57 \\
Al line before [200] & 3.1 & 0.76 \\
Al line after [111] & 1.0 & 1.1 \\
Al line after $[200]$ & 1.3 & 1.4 \\
\hline \hline
\end{tabular}




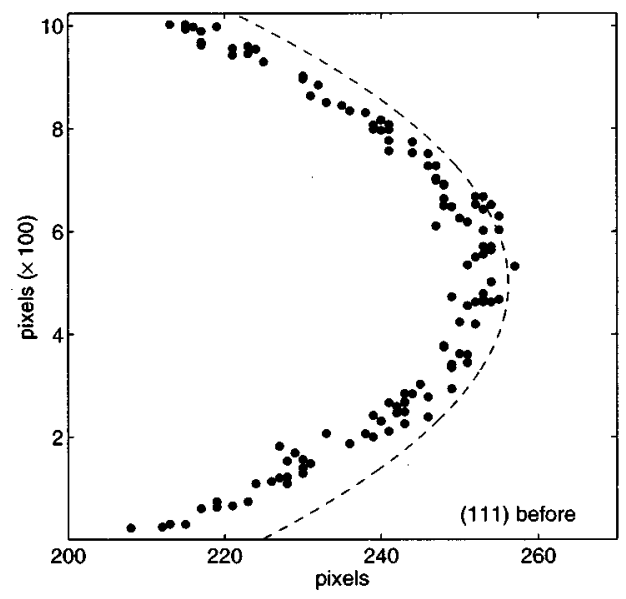

(a)

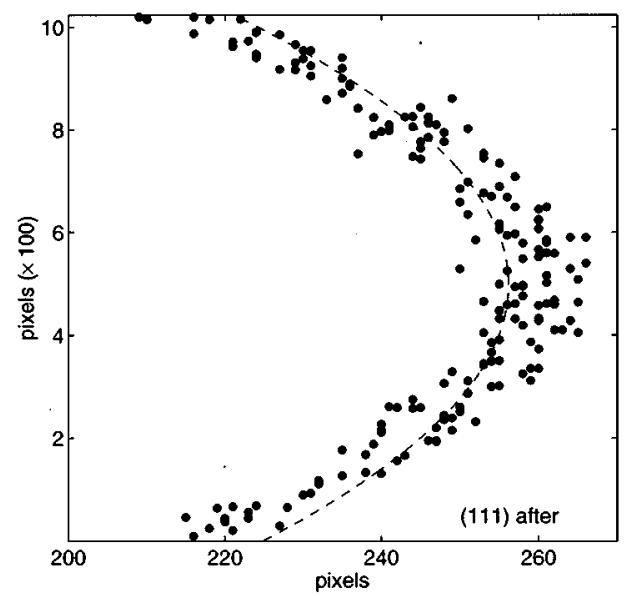

(c)

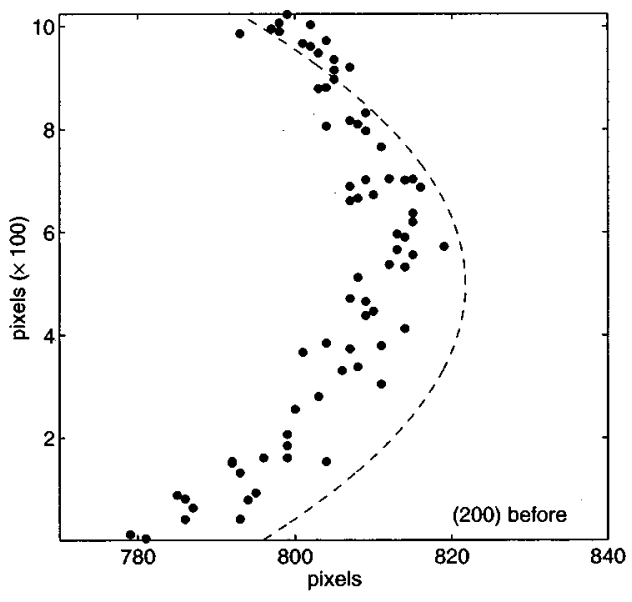

(b)

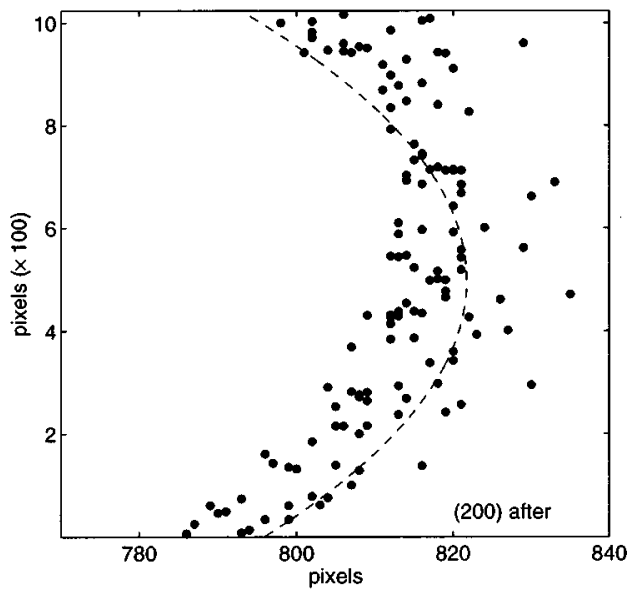

(d)

FIG. 4. Collection of diffraction spots obtained from the patterned line (a)-(b) before and (c)-(d) after electromigration testing. (a), (c) and (b), (d) show the positions of (111) and (200) spots, respectively. The dashed lines mark the calculated diffraction ring positions expected for an unstrained Al powder sample.

the small distance between a diffraction spot and the reference ring is not affected significantly by the distortions.

Table I lists the measured average strain and its standard deviation for the thick film and patterned sample before and after the electromigration testing. The average strain measurements are with respect to the thick film which is assigned a zero average strain. Figure 5 shows the distribution of grain strains in the three samples. The standard deviations for the thick film and the line sample before stressing are similar as well as their distributions shown in Fig. 5 except for a shift in average value. On the other hand, the distribution after accelerated electromigration testing is significantly wider (also evident in Fig. 4) indicating larger variation of strain between the grains.

\section{B. Diffraction spot size analysis}

The position of a diffraction spot on the area detector is related to the strain present in the grain. However, more can be learned about the diffracting crystal (grain) from the size and shape of the individual diffraction spot. In particular, the width of a diffracted beam from a single crystal upon rotation of the crystal around the $w$ axis is a convolution of several terms ${ }^{20}$

$$
\begin{aligned}
w^{2}= & {\left[w_{\text {dyn }}^{2}+\left(\frac{\delta d}{d}\right)_{\text {strain }}^{2}+\left(\frac{\delta \lambda}{\lambda}\right)^{2}\right] \tan \theta^{2} } \\
& +(2 \eta)^{2}+\left(2 \sigma_{\psi}\right)^{2},
\end{aligned}
$$

where $w_{\text {dyn }}$ is the Darwin width of a perfect crystal, $\delta d$ is the variation in lattice spacing in the illuminated sample volume, $\delta \lambda$ is the spectral width of the incident radiation, $\theta$ is the Bragg angle, $\eta$ is the root-mean-square of the mosaic spread of the crystal, and $\sigma_{\psi}$ is the standard deviation of the divergence of the incident beam. The Darwin width term is based on the assumption of a large crystal diffracting in the dynamical mode and needs to be substituted by a crystal size dependent term for smaller crystals diffracting in the kinematic mode. However, this necessary substitution does not concern us for our present purpose. We can separate the broadening of the spot due to the nonuniform strains in the form 


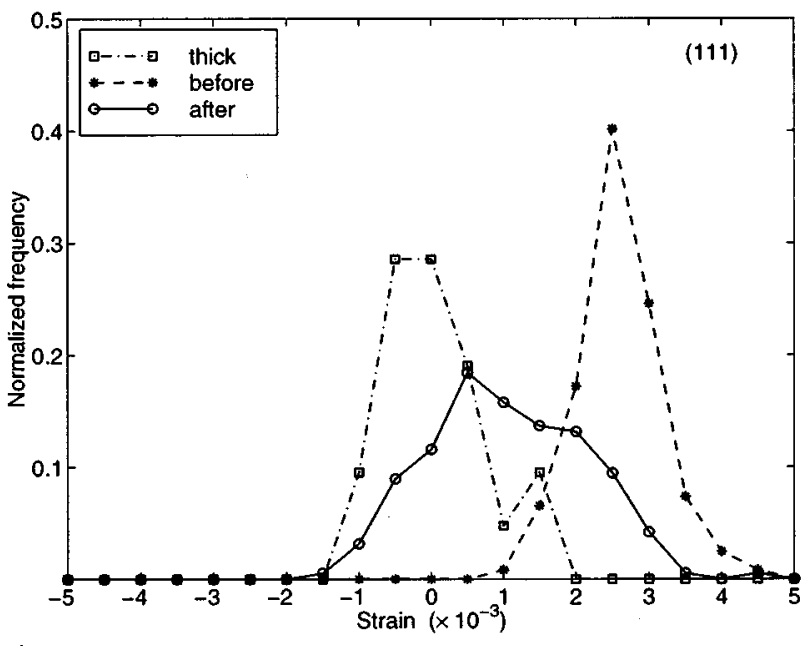

(a)

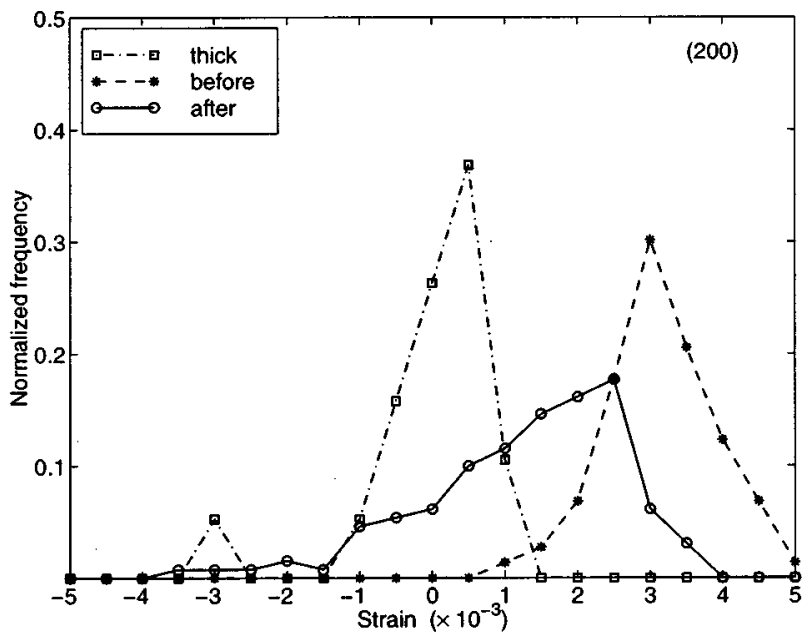

(b)

FIG. 5. Normalized histograms of grain strain measurements for the three samples: thick Al-Cu film, patterned line before and after electromigration testing, (a) (111), (b) (200) grains. The histogram for the line after testing is markedly different from the other two in terms of shape and width due to the larger strain variation in this sample.

$$
w^{2}=w_{0}^{2}+w_{\epsilon}^{2},
$$

where $w_{\epsilon}$ is the broadening due to the nonuniform strain and $w_{0}$ is due to all other effects combined. The contribution of the nonuniform strain is given by the term

$$
w_{\epsilon}^{2}=\left(\frac{\delta d}{d}\right)^{2} \tan ^{2} \theta .
$$

Table II summarizes the spot width measurements obtained in this experiment for the three cases examined. Diffracted beams due to (200) grains consistently have larger width in all three cases. The average spot widths for the thick film and the patterned line before electromigration are practically the same. However, a significant increase in the width is observed after the electromigration testing. We examine Eq. 2 to determine the possible factors contributing to the observed increase in spot width. First, the broadening terms due to incident beam properties are eliminated since these were kept constant throughout the experiment. We do not expect the grain size and the mosaic spread to change under the accelerated test conditions since the samples were annealed at higher temperatures before. Therefore, we attribute the increase in average spot width to the increase in the intragrain strain variation. With this approach we calculate the increase in the intra-grain strain variation to be $6 \times 10^{-4}$. This is equal to about half of the inter-grain strain nonuniformity $\left(\sigma_{\epsilon}\right.$ given in Table I which is in the 1.1-1.4 $\times 10^{-3}$ range).

\section{Strain profile}

We have presented only average and statistical behavior of the microdiffraction data up to this point. Figure 6 shows the variation of the strain along the line as measured by both (111) and (200) reflections. Several (111) and (200) diffraction spots were obtained from most of the inspected points on the line although none were recorded at some positions.
We calculated the average strain at a particular position on the line as the average of the individual grain strains measured at that point. This was done separately for (111) and (200) grains.

The strain profile "before electromigration" is determined largely by grain to grain variation. In order to confirm this statement, two statistical tests were applied to the data. First, if there are position dependent variations of the strain along the line then the strains measured from grains at one experimental point should on the average has smaller variation than the overall one calculated for the whole line. For this purpose the standard deviation of strains for grains was calculated for each point on the line. The average of these values was compared with the standard deviation of strain for all the grains obtained from the particular sample. The results showed that the two averages were practically equal for the before electromigration measurements. In other words, the strains between neighboring grains were not correlated any more than distant ones. Same calculation for the after electromigration measurements indicated that the standard deviation of strain at a particular point was on the average $35 \%-75 \%$ lower than the overall standard deviation. This result strongly indicated that while there was no position specific behavior of strain present before the electromigration test, such was the case after the test.

TABLE II. Average widths of diffraction spots in the $2 \theta$ direction.

\begin{tabular}{lc}
\hline \hline & $w\left(\times 10^{-4} \mathrm{rad}\right)$ \\
\hline Thick Al [111] & 8.5 \\
Thick Al [200] & 8.8 \\
Al line before [111] & 8.7 \\
Al line before [200] & 8.8 \\
Al line after [111] & 10.0 \\
Al line after [200] & 10.7 \\
\hline \hline
\end{tabular}




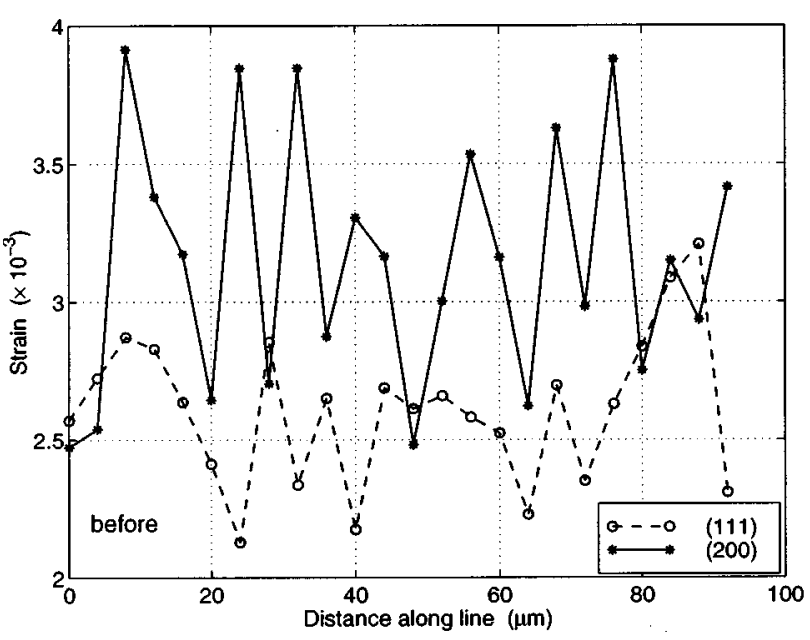

(a)

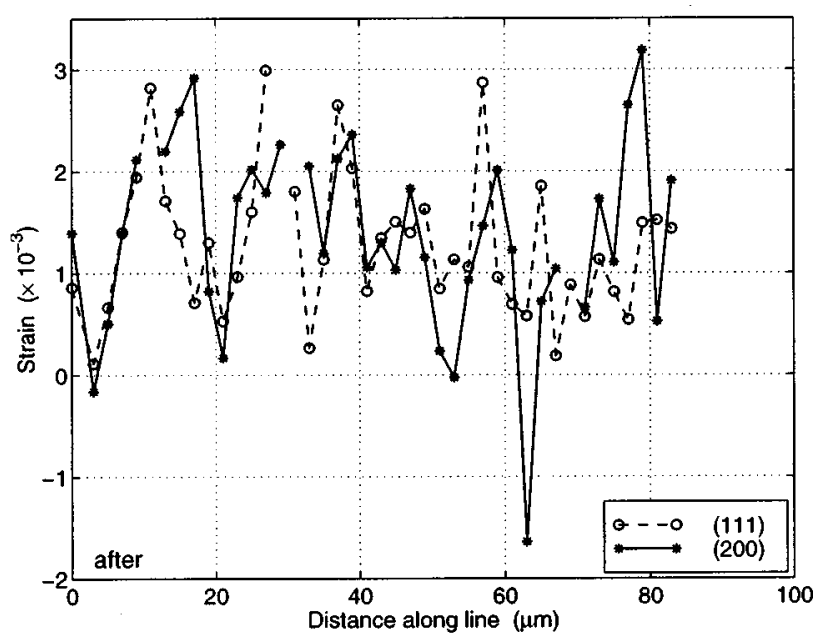

(b)

FIG. 6. Strain profile along line (a) before and (b) after electromigration stressing. Dashed and solid lines show the measurement from (111) and (200) spots, respectively.

Second, again if there are position dependent variations of the strain along the line then the strains measured from the (111) and (200) grains at a position on the line should be correlated. The correlation coefficient between (111) and (200) measurements of strain was -0.15 and 0.34 for the before and after electromigration data respectively. This results indicate correlation of the (111) and (200) measurements after the electromigration test as we also notice in the plots of Fig. 6(b).

It is interesting to note that even though there is a correlation between the stress behavior of (111) and (200) type grains, there are some marked and potentially significant differences. Both the inter and intra-grain strain variations are higher for the grains with (200) orientation. The strain profile in Fig. 6 also shows that the extremes of the (200) profile deviate farther away from the average than the (111) profile. This behavior may be related to the better electromigration resistance of lines with high (111) crystallographic textures. From our results we can expect the strain (and the stress) profile to be smoother in a line with high (111) texture. It is commonly accepted that the failure of an interconnect line is related to the maximum stress level attained by the line. Our results suggest that (111) textured lines with smaller stress variation would be less likely to attain this stress level and consequently would be less likely to fail.

\section{Stress determination}

The general stress state in a solid is given by six independent stress components, three normal and three shear ones. We can define the sample coordinate system for the Al line samples with the axes being parallel to the line length $\left(x_{s}\right)$, width $\left(y_{s}\right)$ and the surface normal $\left(z_{s}\right)$. Average shear strains vanish in this coordinate system due to symmetry. However, shear components of microstresses which are of concern to us may be finite even though their averages are zero. Therefore, in general, six independent measurements of stress is necessary to determine the full stress state.
When the line width is much larger than the film thickness the stress in the line can be assumed to be equi-biaxial. In that case measurement of strain in only one direction becomes sufficient for calculating the in-plane stress, which is the only one present. Besser and Bravman ${ }^{15}$ have made three-dimensional average stress measurements on lines with aspect ratios similar to our samples and have found the stress to be strongly triaxial. Measurements along at least three directions is necessary for specification of the triaxial stress. In our experiments only one strain component, that along the surface normal direction $(\psi=0)$ was measured. This is not sufficient for determining the stress state of our patterned line samples which is expected to have triaxial stress. Moreover the fact that microstresses may have nonzero shear components further complicates the problem requiring at least six measurements.

\section{CONCLUSIONS}

We have measured the strain distribution along an $\mathrm{Al}-\mathrm{Cu}$ line with a $\sim 1 \mu \mathrm{m}$ resolution using x-ray microdiffraction for the first time. The experiment was performed before and after an accelerated testing for electromigration. Significant differences were found in the stress state of the line between before and after electromigration conditions.

In the measurements made before the accelerated testing the grain to grain variation of strain dominated the profile. Whereas in the measurements made after the testing a position dependence of strain was found. This was evidenced by the correlation of strains of neighboring grains and the correlation of the (111) and (200) type grain strains.

(111) and (200) grains exhibit the same general behavior in terms of averages and variation along the line. However, (200) grains exhibit larger deviations from the average. Lines with good (111) crystallographic texture are known to have better electromigration performance. Our results suggest that the (200) grains present in the line may cause attainment of 
higher stresses on the line which may eventually cause failure.

A potential short coming of the present measurements is the time delay between the accelerated testing and the subsequent X-ray measurements. It is known that stresses in patterned Al films substantially relax in several tens of minutes. Therefore, we can expect the actual stresses and their variations to be substantially higher during the test than what our measurements indicate. We are extending our studies to include in situ micro-X-ray diffraction measurements for that purpose.

Determination of stress from strain measurements in fine lines require at least three independent measurements of strain in three directions due to the triaxial stress state of such patterns. Such measurements with micron scale spatial resolution present strong challenges as has been discussed earlier. Work is under way to this end utilizing strong texture properties of $\mathrm{Al}$ alloy lines and predetermination of grain crystallographic orientations with electron microscopy.

\section{ACKNOWLEDGMENT}

Use of the Advanced Photon Source was supported by the U.S. Department of Energy, Basic Energy Sciences, Office of Energy Research under Contract No. W-31-109-Eng38 .
${ }^{1}$ J. Curry, G. Fitzgibbon, Y. Guan, R. Muollo, G. Nelson, and A. Thomas, Proceedings of the 20th Annual International Reliability Symposium (1984), p. 6.

${ }^{2}$ J. Klema, R. Pyle, and E. Domangue, Proceedings of the 20th Annual International Reliability Symposium (1984), p. 1.

${ }^{3}$ R. S. Sorbello, Mater. Res. Soc. Symp. Proc. 225, 3 (1991).

${ }^{4}$ I. A. Blech and H. Sello, Physics of Failures in Electronics, USAF-RADC Series, Vol. 5 (1966), p. 496.

${ }^{5}$ I. A. Blech, J. Appl. Phys. 47, 1203 (1976).

${ }^{6}$ M. A. Korhonen, P. Borgesen, K. N. Tu, and C. Li, J. Appl. Phys. 73, 3790 (1993).

${ }^{7}$ C. L. Bauer and W. W. Mullins, Appl. Phys. Lett. 61, 2987 (1992).

${ }^{8}$ R. J. Gleixner and W. D. Nix, J. Appl. Phys. 83, 3595 (1998).

${ }^{9}$ Y. J. Park and C. V. Thompson, J. Appl. Phys. 82, 4277 (1997).

${ }^{10}$ Y. Liu, C. L. Cox, and R. J. Diefendorf, J. Appl. Phys. 83, 3600 (1998).

${ }^{11}$ Q. Ma, S. Chiras, D. R. Clarke, and Z. Suo, J. Appl. Phys. 78, 1614 (1995).

${ }^{12}$ R. S. Hemmert and M. Costa, in Proceedings of the 29th Annual International Reliability Physics Symposium (IEEE, New York, 1991), p. 64.

${ }^{13}$ P. R. Besser, S. Brennan, and J. C. Bravman, J. Mater. Res. 9, 13 (1994).

${ }^{14}$ P. A. Flinn and C. Chiang, J. Appl. Phys. 67, 2927 (1990).

${ }^{15}$ P. R. Besser and J. C. Bravman, AIP Conf. Proc. 305, 46 (1993).

${ }^{16}$ P.-C. Wang, G. S. Cargill, I. C. Noyan, and C.-K. Hu, Appl. Phys. Lett. 72, 1296 (1998).

${ }^{17}$ W. Yun, B. Lai, D. Shu, A. Khounsary, Z. Cai, J. Barraza, and D. Legnini, Rev. Sci. Instrum. 67, 3373 (1996).

${ }^{18}$ B. Lai et al., Appl. Phys. Lett. 61, 1877 (1992).

${ }^{19}$ A. Krasnoperova et al., J. Vac. Sci. Technol. B 11, 2588 (1993).

${ }^{20}$ P. Coppens, Synchrotron Radiation Crystallography (Academic, London, 1992). 